\title{
A SATISFAÇÃO DA PESSOA COM RETENÇÃO URINÁRIA NO USO DE DIFERENTES TIPOS DE CATETERES VESICAIS
}

\author{
Andreia Bonello ${ }^{1, *} \mathbb{D}$, Mirelle Cristina Faci ${ }^{1}$, Marthyna Pereira de Mello2 (D)
}

\section{RESUMO}

Objetivo: avaliar a satisfação da pessoa com retenção urinária diante do uso de diferentes tipos de cateteres vesicais bem como comparar e analisar diferentes atributos desses após a sua utilização no cateterismo intermitente limpo (CIL). Método: estudo exploratório descritivo de corte transversal, desenvolvido em um ambulatório de referência do estado de São Paulo, no período de fevereiro a março de 2020. A amostra constituiu-se de 32 adultos cadastrados no serviço, e que adotavam o CIL como forma de esvaziamento vesical. A coleta de dados foi realizada por entrevista individual com a utilização de um questionário semiestruturado. Os dados foram analisados por estatística descritiva, inferencial e categorização das respostas para as questões abertas. Resultados: dos 32 usuários, $84 \%$ eram do sexo masculino, com média de idade de 54,16 anos, $81 \%$ realizavam o procedimento entre 1 a 5 anos, com frequência de 4 a 6 vezes ao dia (66\%). A maioria dos entrevistados (73\%) preferiu o cateter de poliuretano com revestimento hidrofílico, convergindo com o percentual de usuários satisfeitos com o mesmo cateter. Conclusão: a tecnologia com maior satisfação é o cateter de poliuretano com revestimento hidrofílico devido a diversos atributos, como lubrificação, firmeza, embalagem, deslizamento na uretra, manipulação, eliminação rápida da urina e conforto.

DESCRITORES: Cateterismo urinário. Bexiga urinária neurogênica. Autocuidado. Educação em saúde. Estomaterapia.

\section{THE SATISFACTION OF THE PERSON WITH URINARY RETENTION IN THE USE OF DIFFERENT TYPES OF URINARY CATHETERS}

\begin{abstract}
Objective: to assess the satisfaction of the person with urinary retention in the face of the use of different types of urinary catheters, as well as to compare and analyze different attributes of these after their use in clean intermittent catheterization (CIC). Method: descriptive exploratory cross-sectional study, developed in a reference clinic in the state of São Paulo, from February to March 2020. The sample consisted of 32 adults registered in the service, and who adopted the CIC as a form of bladder emptying. Data collection was performed through individual interviews using a semi-structured questionnaire. Data were analyzed using descriptive, inferential statistics and categorization of responses to open questions. Results: of the 32 users, $84 \%$ were male, with a mean age of 54.16 years, $81 \%$ performed the procedure between 1 and 5 years, with a frequency of 4 to 6 times a day (66\%). Most respondents (73\%) preferred the hydrophilic-coated polyurethane catheter, converging with the percentage of users satisfied with the same catheter. Conclusion: the most satisfying technology is the
\end{abstract}

1. Faculdades Integradas Einstein de Limeira - Enfermagem em Saúde Coletiva - Limeira (SP), Brasil.

2. Pontifícia Universidade Católica do Paraná - Programa de Pós-graduação em Tecnologia em Saúde - Curitiba (PR), Brasil. *Autora correspondente: acbonello@hotmail.com.br

Editor de Seção: Gisela Maria Assis

Recebido: Maio 2021, 22 | Aceito: Set. 2021, 06

Como citar: Bonello A; Faci MC; Mello MP. A satisfação da pessoa com retenção urinária no uso de diferentes tipos de cateteres vesicais. ESTIMA, Braz. J. Enterostomal Ther., 2021, 19: e2321. https://doi.org/10.30886/estima.v19.1099_PT 
hydrophilic-coated polyurethane catheter due to several attributes such as lubrication, firmness, packaging, urethral slip, handling, rapid urine clearance and comfort.

DESCRIPTORS: Urinary catheterization. Neurogenic urinary bladder. Self-care. Health education. Stomatherapy.

\section{LA SATISFACCIÓN DE LA PERSONA CON RETENCIÓN URINARIA EN EL USO DE DIFERENTES TIPOS DE CATÉTERES VESICALES}

\section{RESUMEN}

Objetivo: evaluar la satisfacción de la persona con retención urinaria ante el uso de diferentes tipos de catéteres vesicales, así como comparar y analizar diferentes atributos de estos después de su utilización en el cateterismo intermitente limpio (CIL). Método: estudio exploratorio descriptivo de corte transversal, desarrollado en un ambulatorio de referencia del estado de San Pablo, en el período de febrero a marzo del 2020. La muestra se constituye de 32 adultos registrados en el servicio, y que adoptaban el CIL como forma de vaciamiento vesical. La recogida de datos fue realizada por entrevista individual con la utilización de un cuestionario semiestructurado. Los datos fueron analizados por estadística descriptiva, inferencial y categorización de las respuestas para las preguntas abiertas. Resultados: de los 32 usuarios, 84\% eran del sexo masculino, con promedio de edad de 54,16 años, 81\% realizaban el procedimiento entre 1 a 5 años, con frecuencia de 4 a 6 veces al día (66\%). La mayoría de los entrevistados (73\%) prefirió el catéter de poliuretano con revestimiento hidrófilo, convergiendo con el porcentual de usuarios satisfechos con el mismo catéter. Conclusión: la tecnología con mayor satisfacción es el catéter de poliuretano con revestimiento hidrófilo debido a diversos atributos, como lubricación, firmeza, embalaje, deslizamiento en la uretra, manipulación, eliminación rápida de la orina y comodidad.

DESCRIPTORES: Cateterismo urinario. Vejiga urinaria neurogénica. Autocuidado. Educación en salud. Estomaterapia.

\section{INTRODUÇÃO}

Algumas doenças no sistema nervoso podem afetar e inviabilizar ou impedir o esvaziamento regular da bexiga, favorecendo retenção urinária parcial, total, temporária ou definitiva, como acontece nos casos de pacientes que vivenciam problemas neurológicos ou idiopáticos do trato urinário inferior ${ }^{1}$.

A forma mais simples e segura de tratar o esvaziamento incompleto da bexiga é o cateterismo intermitente limpo (CIL), considerado o padrão ouro, com frequência de 4 a 6 vezes por dia. O procedimento é feito entre 16 a $56 \%$ da população com lesão medular espinhal, visando melhorar a funcionalidade da bexiga e proteger a parte superior do trato urinário, além do impacto positivo na qualidade de vida, mas paradoxalmente a consequência mais proeminente ainda é a recidiva de infecções do trato urinário ${ }^{2-4}$.

Trata-se de uma técnica simples em que o próprio usuário ou seu cuidador introduz o cateter na bexiga, através da uretra ou conduto cateterizável, sem uso de antissépticos ou luvas estéreis. O procedimento foi descrito em 1970 por Jack Lapides e colaboradores, que demonstraram que a prevenção da infecção urinária nesses pacientes se dá pela prevenção da distensão vesical e eliminação do resíduo e não pela assepsia da técnica. Daí vem o nome de técnica limpa e não estéril ${ }^{5,6}$.

$\mathrm{Na}$ categoria de Enfermagem, o responsável por realizar a capacitação do CIL é o enfermeiro generalista habilitado ou com especialização em estomaterapia ou em reabilitação, sendo o profissional capacitado para cuidar com destreza e segurança desse perfil de paciente, seja no âmbito hospitalar, ambulatorial e na assistência domiciliar. Assim como atuam na capacitação dos pacientes a respeito da melhor conduta e tecnologias existentes para a prática do CIL, favorecendo sua independência e melhor qualidade de vida ${ }^{7,8}$.

Atualmente, no cenário nacional e mundial existem diferentes tipos de cateteres vesicais para a realização do CIL. Há um número crescente de publicações comparando tais tecnologias, dada a importância do tema. Enfatiza-se ainda que 
a indicação do cateter mais adequado à necessidade e preferências do cliente é um fator determinante para sua adesão à técnica ${ }^{9,10}$.

No Brasil, os usuários do cateterismo urinário intermitente não possuem, até o momento, política específica que garanta os recursos necessários para atendimento de excelência. O paciente é atendido como todo cidadão usuário dependente do Sistema Único de Saúde (SUS), tem direito aos materiais necessários ao procedimento, mas nem sempre esses são obtidos ${ }^{9}$.

Os cateteres mais frequentemente utilizados são os de polivinilcloreto (PVC) não revestidos, que necessitam da aplicação manual de lubrificante para facilitar a introdução e reduzir a fricção. Quando se compara tal realidade a de outros países, observam-se mudanças nessas políticas, as quais parecem apoiar com maior intensidade o uso de tecnologias mais avançadas na realização do procedimento ${ }^{4,9}$.

Diante do cenário exposto, considerando o fato do CIL ser o padrão ouro de esvaziamento para retenção urinária, por existirem tecnologias mais avançadas que favorecem a adesão ao procedimento e, consequentemente, impactam na qualidade de vida e autonomia dos pacientes como também na redução dos riscos de complicações associadas ao procedimento, considera-se relevante analisar a satisfação dos usuários no uso de diferentes cateteres vesicais.

Assim, apresenta-se como objetivo deste estudo: avaliar a satisfação da pessoa com retenção urinária em relação aos diferentes tipos de cateteres vesicais, e comparar e analisar os diferentes atributos desses após a sua utilização no CIL.

\section{MÉTODO}

Trata-se de um estudo exploratório descritivo de corte transversal e com abordagem quali-quantitativa, envolvendo usuários com retenção urinária cadastrados em um Ambulatório de Feridas, Incontinência e Estomias do Estado de São Paulo.

Como critérios de inclusão estabeleceram-se: usuários cadastrados no serviço, maiores de 18 anos, de ambos os sexos, com retenção urinária e que realizavam o CIL como forma de esvaziamento vesical, independente do tempo de início do procedimento, além de estarem cadastrados no referido ambulatório e terem vínculo com o local de coleta, autonomia na realização do autocateterismo e utilização de cateter com calibres no10 ou nº12 fornecido pelo serviço de saúde.

Foram excluídos os usuários que apresentavam contraindicação para uso das tecnologias do estudo, que não realizavam a autocateterização, apresentando dependência de terceiros (familiar, cuidadores, amigos e outros) e que não utilizava

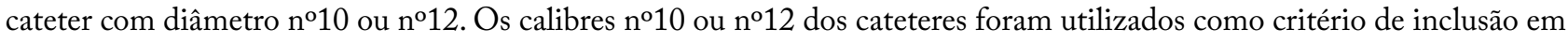
decorrência de serem produzidos por todas as empresas inseridas na pesquisa.

Do total de 88 pacientes com retenção urinária cadastrados no serviço, 65 faziam uso de cateter com calibres no10 ou n¹2, porém apenas 32 pacientes se enquadraram em todos os critérios de inclusão. Todos maiores de 18 anos, com retenção urinária e que realizavam o CIL como forma de esvaziamento vesical, cadastrados no serviço, usando cateter com calibres $\mathrm{n}^{\circ} 10$ ou nº12, realizavam o autocateterismo, 27 pacientes (84\%) do sexo masculino.

$\mathrm{Na}$ etapa de pré-coleta, os usuários incluídos no estudo foram randomizados de forma que cada pesquisador ficasse responsável pela orientação, acompanhamento e coleta de informações de quatro pacientes. Os pesquisadores organizaram os "kits pesquisa" compostos pelos cateteres incluídos no estudo em número suficiente para a utilização por 24 horas, devido ao quantitativo recebido por intermédio de doação das empresas fabricantes e respeitando o intervalo recomendado entre as cateterizações; a instrução de uso de cada cateter; o formulário "passo a passo"; o formulário de "anotação"; além do agendamento de retorno para o terceiro momento.

Ainda nessa fase, houve um treinamento teórico e outro prático, totalizando 16 horas distribuídas em 4 tardes, realizados com o apoio das empresas e com a participação de todos os membros inseridos no projeto, com o objetivo de alinhar o conhecimento sobre o tema e as particularidades de cada cateter usado no estudo.

Cada participante avaliou quatro cateteres vesicais de diferentes tecnologias, fornecidos de acordo com os seus cadastros no serviço, respeitando o número de cateterismos realizados no dia, seguindo uma sequência de uso determinada por randomização, conduzida pelo pesquisador juntamente com o responsável pela unidade de saúde, o qual teve participação espontânea, e na presença dos participantes envolvidos no estudo.

A sequência de uso dos cateteres se deu da seguinte forma: 
Dia 1 - Cateter uretral, confeccionado em PVC, provido de substância com propriedades lubrificantes, da BbraunActreen Lite Cath, respectiva marca e modelo.É um cateter pré-lubrificado à base de água e glicerina, de uso único, estéril. Disponível nas versões masculina e feminina.

Dia 2 - Cateter uretral com revestimento hidrofílico, confeccionado em poliuretano, provido de substâncias com propriedades lubrificantes à base de polivinilpirrolidona e instantaneamente pronto para uso, da Coloplast - SpeediCath Standard, disponível nas versões masculina, feminina e pediátrica.

Dia 3 - Cateter uretral, confeccionado de elastômeros de poliolefinas (Carbono, Hidrogênio e Oxigênio), em que a embalagem mantém a água separada do cateter até a ativação, da WellSpect - Lofric Primo, disponível nas versões masculina, feminina e pediátrica.

Dia 4 - Cateter uretral convencional de PVC, sem lubrificação, de uso único, atóxico, flexível, transparente, da Embramed.

Vale destacar que dos cateteres envolvidos na pesquisa, apenas dois eram padronizados e fornecidos pelo serviço regularmente: o cateter uretral com revestimento hidrofilico, confeccionado em poliuretano e pronto para uso, e o cateter uretral convencional de PVC sem lubrificação.

A pesquisa foi desenvolvida em três momentos: primeiro, segundo e terceiro. No primeiro momento, os indivíduos foram informados que o presente estudo era parte de um Trabalho de Conclusão de Curso de Graduação em Enfermagem, submetido ao Comitê de Ética em Pesquisa das Faculdades Integradas Einstein de Limeira - (CEP - FIEL) e aprovado sob o número 3.785.429, e assinaram o Termo de Consentimento Livre e Esclarecido, de acordo com a Resolução CNS/ MS466/12, após esclarecimentos sobre o objetivo do estudo e aceite em participar, no momento da entrevista individual

Em seguida receberam orientações dos próprios pesquisadores sobre a utilização de cada um dos cateteres, o cronograma e sequência de uso, levando para casa um "kit pesquisa"; o formulário "passo a passo"; o formulário de "anotação"; e o agendamento de retorno para o terceiro momento - entrevista.

No segundo momento, os pacientes, em seu domicílio, realizaram o CIL, seguindo sua rotina cotidiana, fazendo o uso dos diferentes tipos de cateteres incluídos na pesquisa, de acordo com a sequência estabelecida, adotando as orientações recebidas dos pesquisadores, no primeiro momento, bem como as orientações escritas disponibilizadas no interior do "kit pesquisa”. Ao final de cada dia, após terem feito todos os procedimentos de cateterismos usando o cateter determinado para uso no dia, fizeram o registro das especificidades do dispositivo no formulário "anotação" com a finalidade de registrar sua percepção.

No terceiro momento, depois do período para a utilização dos diferentes cateteres, os indivíduos retornaram ao local de estudo, ou conforme necessidade, os envolvidos no projeto foram ao domicílio do usuário, em data pré-agendada no momento da entrega dos cateteres (primeiro momento) para responderem às questões do instrumento de pesquisa "satisfação" por meio da entrevista que foi realizada pelos pesquisadores. Neste momento foi recolhido também os formulários "anotação", com o registro das especificidades de cada um dos dispositivos.

Aqueles que por algum motivo não conseguiram desenvolver as ações pertinentes aos momentos da pesquisa foram orientados a retornar à unidade de saúde em data agendada para realização de nova entrevista. Caso a situação permanecesse, esses estariam classificados como "impossibilitado para o procedimento de pesquisa”, sendo, então, excluídos do estudo no seu percurso. Não houve nenhum impedimento de participação pelo motivo em questão.

O kit pesquisa incluiu o quantitativo de cateteres contemplados no estudo suficientes para utilização em 24 horas, segundo o cadastro no serviço, de acordo com a frequência de cateterismos realizados pelo paciente em um dia.

A coleta de dados ocorreu por meio de entrevista individual com os usuários no serviço de saúde ou no seu próprio domicílio, concretizada no período de fevereiro a março de 2020, sendo realizada às segundas e terças-feiras, no horário das 13 às $17 \mathrm{~h}$ ou de acordo com a necessidade do serviço e disponibilidade dos pesquisadores envolvidos.

Foi utilizado como referência para a construção do instrumento de coleta, um questionário semiestruturado já validado no Brasil: "Instrumento de Avaliação da Percepção do Usuário na Utilização de Cateteres Vesicais"11.

O referido questionário foi construído pelos pesquisadores e profissionais de enfermagem do serviço com a participação da enfermeira estomaterapeuta da referida unidade. Buscando uma boa interpretação e entendimento do instrumento elaborado, foi realizado um estudo piloto em três etapas, com os membros da pesquisa, funcionários da unidade de saúde 
e usuários cadastrados no serviço, permitindo, dessa maneira, fazer ajustes para melhor compreensão do entrevistado e do entrevistador antes da sua aplicação.

As questões fechadas apresentavam alternativas a serem escolhidas pelos usuários no momento da entrevista, já as questões abertas foram respondidas de acordo com a expectativa e a percepção do entrevistado. $\mathrm{O}$ instrumento de coleta de dados contempla aspectos relacionados aos quatro cateteres, incluindo a abertura da embalagem, a manipulação, o seu deslizamento na uretra, a sua retirada, o tempo de procedimento, a percepção do tempo de procedimento e pontos positivos e negativos de cada tecnologia avaliada.

As questões "abertura da embalagem", "manipulação", "deslizamento na uretra", "retirada”, "tempo de procedimento" e "percepção do tempo de procedimento" foram respondidas em uma escala do tipo Likert de cinco pontos, e as respostas são de "muito fácil" a "muito difícil", "muito bom" a "muito ruim" ou "muito rápido" a "muito demorado", respectivamente pontuandos de 1 a 5, ou seja, quanto menor a pontuação, maior é a satisfação no uso. Foi considerado como satisfeito o sujeito participante que assinalasse as respostas: "fácil" e "muito fácil" ou "bom” e "muito bom", tendo em vista que o "razoável" ou nota três é a linha de corte entre satisfação e insatisfação. Em relação ao tempo de procedimento foi considerado dentro dos padrões esperados "adequado" o sujeito que mencionasse as respostas "muito rápido" e "rápido", tendo em vista que o "razoável" ou nota três é a linha de corte entre o esperado e o real. Foi, também, solicitado ao participante que ele mencionasse pontos positivos e negativos referentes a cada cateter analisado. No término do questionário foi indagado ao cliente qual das tecnologias atendeu melhor às suas expectativas e por qual motivo.

A análise estatística foi realizada através do software SPSS Statistics (versão 23), a comparação dos cateteres foi realizada utilizando-se o teste não paramétrico de Mann-Whitney, em que foi considerada significância estatística para valores de $(\mathrm{P}<0,05)$. Já na apreciação dos dados qualitativos, para as questões abertas foram criadas duas categorias: "pontos positivos" e "pontos negativos", a partir da leitura prévia de todo o conteúdo do questionário respondido e agrupamento por similaridade de resposta. A categoria "pontos positivos” foi considerada quando, na nossa percepção, a pessoa entrevistada apontava alguma característica positiva em relação ao aspecto abordado. Já a categoria "pontos negativos" foi acatada quando, na nossa percepção, o entrevistado apontava alguma característica negativa em relação ao aspecto abordado.

\section{RESULTADOS}

No total, foram abordados 32 pacientes com retenção urinária cadastrados em um ambulatório de referência do Estado de São Paulo, sendo 84\% da amostra de predominância do sexo masculino. A Tabela 1 apresenta os dados do perfil da amostra que teve como predominância idade superior a 60 anos (40,63\%) e idade média da amostra total de 54,16 anos. A totalidade dos cadastrados estava vinculada ao serviço há mais de 500 dias. Do total de pacientes, 40,62\% referiu realizar o procedimento no intervalo de tempo de 1.501 a 3.000 dias e $87 \%$ realizam com frequência diária de 4 a 6 cateterismos.

Tabela 1. Distribuição dos pacientes com retenção urinaria entrevistados, segundo perfil demográfico e clínico. Limeira (SP) - 2020.

\begin{tabular}{lc}
\hline \multicolumn{1}{l}{ Variáveis e categorias } & $\mathbf{n}=\mathbf{3 2}(\%)$ \\
\cline { 1 - 2 } Idade & $7(21,88)$ \\
\hline Até 40 anos & $7(21,88)$ \\
\hline 41 a 50 anos & $54,16 \pm 13,65(76-32)$ * anos \\
\hline 51 a 60 anos 60 anos & $13(40,63)$ \\
\hline Total & $32(100,00)$ \\
\hline
\end{tabular}


Tabela 1. Continuação...

\begin{tabular}{|c|c|c|}
\hline Variáveis e categorias & \multirow{2}{*}{$\mathrm{n}=32(\%)$} & \\
\hline Tempo de Cadastro no Programa (Dias) & & \\
\hline Até 500 dias & $6(18,75)$ & \multirow{5}{*}{$2042,31 \pm 1504,97(5400-14) *$ dias } \\
\hline 501 a 1500 dias & $6(18,75)$ & \\
\hline 1501 a 3000 dias & $14(43,75)$ & \\
\hline > 3000 dias & $6(18,75)$ & \\
\hline Total & $32(100)$ & \\
\hline \multicolumn{3}{|l|}{ Tempo de CIL (Dias) } \\
\hline Até 500 dias & $6(18,75)$ & \multirow{5}{*}{$2293,56 \pm 1994,29(9360-14) *$ dias } \\
\hline 501 a 1500 dias & $6(18,75)$ & \\
\hline 1501 a 3000 dias & $13(40,625)$ & \\
\hline > 3000 dias & $7(21,875)$ & \\
\hline Total & $32(100)$ & \\
\hline \multicolumn{3}{|l|}{$\mathrm{N}^{\circ} \mathrm{CIL} / \mathrm{DIA}$} \\
\hline 4 & $7(21,875)$ & \multirow{6}{*}{$5,44 \pm 1,11(8-4) * n^{\circ}$ CIL/dia } \\
\hline 5 & $10(31,25)$ & \\
\hline 6 & $11(34,375)$ & \\
\hline 7 & $2(6,25)$ & \\
\hline 8 & $2(6,25)$ & \\
\hline Total & $32(100)$ & \\
\hline
\end{tabular}

*Média e desvio padrão \pm (máximo-mínimo); CIL = Cateterismo Intermitente Limpo.

A Tabela 2 apresenta os atributos avaliados com valores para média e a diferença estatística entre os cateteres avaliados. Em relação ao quesito abertura da embalagem, a resposta "muito fácil" foi mencionada com maior frequência pelos entrevistados ao avaliarem o cateter de poliuretano (47\%), o que demonstrou diferença estatística significativa quando comparado ao cateter PVC com propriedades lubrificantes ( $\mathrm{p}=0,014)$, ao cateter de elastômeros de poliolefinas $(\mathrm{P}=0,006)$ e ao cateter $\mathrm{PVC}$ convencional $(\mathrm{p}=0,003)$. Vale destacar que, ainda, nesse quesito, o cateter 1 apresentou diferença estatisticamente significativa em relação ao cateter 4 ( $p=0,039$ ), e $28 \%$ dos entrevistados considerou a manipulação do cateter PVC convencional "muito difícil”.

Quanto à percepção sobre a manipulação do cateter, a resposta "muito fácil” foi mencionada por $47 \%$ dos entrevistados ao avaliarem o cateter de poliuretano, apresentando significância quando comparado ao cateter PVC com propriedades lubrificantes $(p=0,002)$, ao cateter de elastômeros de poliolefinas $(p=0,012)$ e ao cateter PVC convencional $(p=0,001)$. A diferença entre o cateter de elastômeros de poliolefinas e de PVC convencional também demonstrou significância $(\mathrm{p}=0,024)$.

Sobre a análise do deslizamento na uretra, a maior parte dos entrevistados (41\%) considerou "fácil" o deslizamento do cateter PVC com propriedades lubrificantes, e a mesma percepção foi encontrada em relação ao cateter de poliuretano, porém por um número maior de pacientes (53\%), havendo significância estatística quando comparado ao cateter PVC com propriedades lubrificantes ( $\mathrm{p}=0,001)$, ao cateter de elastômeros de poliolefinas $(\mathrm{p}=0,002)$ e ao cateter de PVC convencional $(\mathrm{p}=0,000)$. Nessa mesma análise o cateter 1 comparado ao 4 também apresentou relevância estatística $(\mathrm{p}=0,007)$, e o cateter 3 quando comparado ao 4 ( $\mathrm{p}=0,002)$. 
$\mathrm{Na}$ avaliação de retirada da uretra a resposta 'fácil' foi mencionada com maior frequência pelos entrevistados na avaliação de todos os cateteres estudados, sendo o cateter PVC com propriedades lubrificantes (50\%) e com significância quando comparado ao cateter 4 ( $\mathrm{p}=0,008)$. O cateter de poliuretano com revestimento hidrofilico (47\%) e quando comparado ao cateter 1 ( $p=0,015)$ e ao cateter 4 ( $p=0,000)$. Já o cateter elastômero de poliolefinas (41\%), e significância quando comparado com o cateter 4 ( $\mathrm{p}=0,002)$. E finalmente o cateter PVC convencional (28\%), sem relevância quando comparado aos demais.

Já em tempo de procedimento a resposta "muito fácil" foi referida com maior frequência entre os entrevistados ao avaliarem o cateter de poliuretano (81\%), sendo relevante em minutos quando comparado ao cateter 4 ( $p=0,003)$. Para essa mesma análise o cateter 1 quando comparado ao 4 teve significância ( $\mathrm{p}=0,002)$ e o cateter 3 quando comparado ao 4 ( $\mathrm{p}=0,035)$.

Ao se analisar o mesmo quesito, sem considerar os minutos, o cateter de poliuretano também manteve diferença significativa quando comparado ao cateter PVC com propriedades lubrificantes $(p=0,002)$, ao cateter de elastômeros de poliolefinas $(\mathrm{p}=0,002)$ e ao cateter PVC convencional $(\mathrm{p}=0,000)$. Também com diferença significativa entre o cateter PVC com propriedades lubrificantes comparado ao cateter PVC convencional $(\mathrm{p}=0,022)$ e o cateter de elastômeros de poliolefinas em relação ao cateter convencional de PVC $(\mathrm{p}=0,025)$.

Tabela 2. Satisfação dos pacientes com retenção urinaria entrevistados em relação à avaliação de cateteres vesicais de diferentes tecnologias. Limeira (SP) - 2020

\begin{tabular}{|c|c|c|c|c|c|c|c|c|c|c|}
\hline $\begin{array}{c}\text { Atributo } \\
\text { Avaliado } \\
\text { (valores para } \\
\text { média) }\end{array}$ & $\mathrm{C} 1$ & $\mathrm{C} 2$ & C3 & $\mathrm{C} 4$ & $\begin{array}{l}\text { Valor de } \\
\text { P para } \\
\text { diferença } \\
\text { C1 x C2 }\end{array}$ & $\begin{array}{l}\text { Valor de } \\
\text { P para } \\
\text { diferença } \\
\text { C1 x C3 }\end{array}$ & $\begin{array}{l}\text { Valor de } \\
\text { P para } \\
\text { diferença } \\
\text { C1 x C4 }\end{array}$ & $\begin{array}{l}\text { Valor de } \\
\text { P para } \\
\text { diferença } \\
\text { C2 x C3 }\end{array}$ & $\begin{array}{l}\text { Valor de } \\
\text { P para } \\
\text { diferença } \\
\text { C2 x C4 }\end{array}$ & $\begin{array}{c}\text { Valor de } \\
\text { P para } \\
\text { diferença } \\
\text { C3 x C4 }\end{array}$ \\
\hline \multicolumn{11}{|c|}{ Escala de avaliação da percepção do cliente quanto ao cateter no CIL } \\
\hline $\begin{array}{l}\text { Abertura } \\
\text { (01 a 05) }\end{array}$ & 2,4 & 1,8 & 2,6 & 2,8 & 0,014 & 0,212 & 0,039 & 0,006 & 0,003 & 0,464 \\
\hline $\begin{array}{l}\text { Manipulação } \\
\text { (01 a 05) }\end{array}$ & 2,7 & 1,9 & 2,6 & 3,3 & 0,002 & 0,969 & 0,091 & 0,012 & 0,001 & 0,024 \\
\hline $\begin{array}{l}\text { Deslizamento } \\
\text { na Uretra } \\
\text { (01 a 05) }\end{array}$ & 2,4 & 1,6 & 2,3 & 3,3 & 0,001 & 0,540 & 0,007 & 0,002 & 0,000 & 0,002 \\
\hline $\begin{array}{l}\text { Retirada } \\
\text { (01 a 05) }\end{array}$ & 2,2 & 1,7 & 2,0 & 3,0 & 0,015 & 0,257 & 0,008 & 0,061 & 0,000 & 0,002 \\
\hline $\begin{array}{l}\text { Tempo de } \\
\text { Procedimento } \\
\text { em Minutos } \\
\text { (01 a 05) }\end{array}$ & 1,3 & 1,3 & 1,6 & 2,0 & 0,527 & 0,074 & 0,002 & 0,075 & 0,003 & 0,035 \\
\hline Tempo de & & & & & & & & & & \\
\hline $\begin{array}{l}\text { Procedimento } \\
\text { II (01 a 05) }\end{array}$ & 2,2 & 1,5 & 2,3 & 2,9 & 0,002 & 0,804 & 0,022 & 0,002 & 0,000 & 0,025 \\
\hline
\end{tabular}

Teste Não Paramétrico de Wilcoxon, $\mathrm{p}<0,05 ; \mathrm{CIL}=$ Cateterismo Intermitente Limpo; C1 = Cateter polivinilcloreto com propriedades lubrificantes; C2= Cateter de poliuretano com lubrificação hidrofilica; C3= Cateter elastômeros de poliolefinas; C4= Cateter polivinilcloreto convencional.

As questões abertas foram categorizadas em "pontos positivos" e "pontos negativos", conforme Tabela 3. Entre os pontos positivos citados pelos entrevistados em relação à avaliação dos diferentes cateteres estudados, destacaram-se: a facilidade de abertura da embalagem, a boa lubrificação, a firmeza e o fácil deslizamento na uretra, presentes nos cateteres de PVC com propriedades lubrificantes, cateter poliuretano e no cateter elastômeros de poliolefinas. O cateter de PVC com propriedades lubrificantes também recebeu elogios como sendo de "fácil" manipulação, transporte e retirada da uretra. Foi referido que o cateter de poliuretano com revestimento hidrofílico apresenta qualidades como praticidade, manipulação, independência, facilidade e rapidez na eliminação da urina e retirada da uretra. Em relação aos pontos positivos citados do cateter convencional de PVC, destacaram-se: o fato de possuir tampa facilitar o manejo e a facilidade no transporte. 
Tabela 3. Pontos positivos e negativos mencionados pelos pacientes com retenção urinária entrevistados em relação à avaliação dos diferentes cateteres estudados. Limeira (SP) - 2020.

\begin{tabular}{|c|c|c|c|c|}
\hline Categorias & Cateter 1 & Cateter 2 & Cateter 3 & Cateter 4 \\
\hline \multirow{12}{*}{ Pontos positivos } & Lubrificação & Lubrificação & Lubrificação & Praticidade \\
\hline & Embalagem & Embalagem & Embalagem & Embalagem \\
\hline & Firmeza & Firmeza & Firmeza & Possui tampa \\
\hline & $\begin{array}{l}\text { Utilizou a embalagem } \\
\text { como guia }\end{array}$ & $\begin{array}{c}\text { Fácil abertura da } \\
\text { embalagem }\end{array}$ & Bom deslizamento & $\begin{array}{c}\text { Eliminação rápida da } \\
\text { urina }\end{array}$ \\
\hline & Fácil manipulação & Praticidade & & Manejo \\
\hline & Fácil transporte & $\begin{array}{l}\text { Não machuca durante } \\
\text { o procedimento }\end{array}$ & & Fácil transporte \\
\hline & Bom deslizamento & Fácil retirada & & \\
\hline & Fácil retirada & Qualidade do material & & \\
\hline & & $\begin{array}{c}\text { Eliminação da urina } \\
\text { rápida }\end{array}$ & & \\
\hline & & Manipulação & & \\
\hline & & Bom deslizamento & & \\
\hline & & Independência & & \\
\hline \multirow{9}{*}{ Pontos negativos } & Embalagem & Embalagem & $\begin{array}{c}\text { Dificuldade para ativar } \\
\text { a embalagem }\end{array}$ & $\begin{array}{c}\text { Demora no } \\
\text { procedimento }\end{array}$ \\
\hline & $\begin{array}{c}\text { Dificuldade na abertura } \\
\text { da embalagem }\end{array}$ & $\begin{array}{l}\text { Falta de tampa na } \\
\text { extremidade }\end{array}$ & Dificuldade na abertura & $\begin{array}{l}\text { Dor e desconforto } \\
\text { durante procedimento }\end{array}$ \\
\hline & $\begin{array}{c}\text { Dificuldade na retirada } \\
\text { da embalagem }\end{array}$ & $\begin{array}{l}\text { Dificuldade no } \\
\text { transporte }\end{array}$ & $\begin{array}{l}\text { Falta de firmeza } \\
\text { durante o } \\
\text { procedimento }\end{array}$ & Falta de lubrificação \\
\hline & $\begin{array}{c}\text { Falta de firmeza na } \\
\text { sonda durante o } \\
\text { procedimento }\end{array}$ & Falta de guia & $\begin{array}{l}\text { Dificuldade na retirada } \\
\text { da sonda }\end{array}$ & $\begin{array}{l}\text { Dificuldade na } \\
\text { manipulação }\end{array}$ \\
\hline & $\begin{array}{l}\text { Dificuldade na } \\
\text { manipulação }\end{array}$ & Pouca lubrificação & Lubrificação & $\begin{array}{c}\text { Dificuldade na retirada, } \\
\text { da sonda da uretra }\end{array}$ \\
\hline & $\begin{array}{l}\text { Falta de tampa na } \\
\text { extremidade }\end{array}$ & $\begin{array}{c}\text { Falta de disponibilidade } \\
\text { do cateter na rede. }\end{array}$ & $\begin{array}{l}\text { Falta de tampa na } \\
\text { extremidade }\end{array}$ & \\
\hline & $\begin{array}{l}\text { Dificuldade no } \\
\text { transporte }\end{array}$ & & $\begin{array}{l}\text { Dificuldade no } \\
\text { transporte }\end{array}$ & \\
\hline & Falta de guia & & $\begin{array}{l}\text { Comprimento do } \\
\text { cateter pequeno }\end{array}$ & \\
\hline & Pouca Lubrificação & & & \\
\hline
\end{tabular}

Já os pontos negativos do cateter de PVC com propriedades lubrificantes foram a dificuldade de abertura da embalagem e a retirada do cateter, falta de firmeza na manipulação e a falta de tampa no cateter.Já em relação ao cateter de poliuretano foram apontados o fato de a embalagem ser muito grande, falta de guia e tampa no cateter. No cateter de poliolefinas, a abertura e a manipulação da embalagem, a ativação da lubrificação, a falta de tampa e dificuldade no transporte. No cateter convencional foram destacados a demora no procedimento, falta de lubrificação e dificuldade na manipulação e retirada do cateter. 
Em relação à preferência do cateter após a pesquisa, a maior parte dos entrevistados (73\%) referiu preferir o cateter de poliuretano com revestimento hidrofílico pronto para uso, convergindo com o percentual de usuários satisfeitos com o mesmo cateter em todos os aspectos avaliados. A preferência está justificada pela boa lubrificação, firmeza, facilidade de abertura da embalagem, praticidade, o fato de "não machucar", deslizamento na uretra, manejo, manipulação, eliminação rápida da urina e conforto. Vale ressaltar que 20 entrevistados (62,5\%) já utilizam o cateter de poliuretano na prática do CIL.

\section{DISCUSSÃO}

São escassos os estudos que focam a satisfação do usuário, a ênfase maior é em pesquisas que comprovem a eficiência e custo efetividade dos dispositivos. Assim, para discutir os resultados da avaliação realizada foram incorporados trabalhos conduzidos na mesma linha de pesquisa, contudo com diferentes objetivos e métodos traçados.

Estudo que buscou avaliar a satisfação de pessoas com lesão medular em relação à utilização de cateter convencional, hidrofílico e pré-lubrificado com bolsa na realização de CIL mostrou resultados semelhantes ao deste trabalho, apontando que o cateter hidrofilico foi considerado superior ao convencional para abertura da embalagem e deslizamento na inserção. O cateter pré-lubrificado com bolsa foi superior ao convencional em abertura da embalagem, introdução, deslizamento e retirada. Já a avaliação do cateter convencional foi divergente ao deste estudo, uma vez que citou como ponto positivo do cateter de PVC o fato de possuir tampa facilitar o manejo e o transporte ${ }^{12}$.

Quando o cateter com revestimento hidrofilico de poliuretano foi comparado com o cateter convencional e o cateter de PVC lubrificado em relação à satisfação do usuário em pesquisa realizada, ele ganhou destaque no quesito abertura de embalagem, o que foi também demostrado nos dados obtidos neste trabalho ${ }^{12}$.

Pesquisa apontou resultados semelhantes ao deste estudo ao concluir que a redução de risco para o desenvolvimento de infecções do trato urinário (ITU), facilidade de inserção e conveniência são os atributos mais apreciados para cateteres intermitentes entre os pacientes ${ }^{13}$.

Outra metanálise recente demonstrou as vantagens dos cateteres hidrofílicos na redução do risco de ITU e trauma uretral, além da satisfação do paciente ${ }^{14}$.

Resultados de um ensaio clínico controlado e randomizado comparando cateteres hidrofilicos com não revestidos, em crianças com bexiga neurogênica, também evidenciou aspectos semelhantes ao deste estudo sobre a satisfação em relação ao cateter hidrofilico, contudo com algumas ressalvas, de acordo com o perfil do usuário, sobre o revestimento escorregadio de difícil manuseio ${ }^{15}$.

Outro estudo com resultado análogo ao desta pesquisa mostra ao identificar as principais dificuldades relatadas pelos pacientes e cuidadores na prática do CIL que a inserção do cateter, a dor, o desconforto, o trauma uretral, banheiros públicos com instalações inadequadas, dificuldades físicas e falta de acesso aos insumos necessários constituem aspectos relacionados ao grau de satisfação e à adesão aos programas de reabilitação vesical, com impacto na qualidade de vida dos pacientes ${ }^{16}$.

Alguns pacientes consideram que o tempo gasto com preparo dos materiais e a realização do procedimento são fatores de dificuldade na realização e adesão ao CIL, por alterarem a rotina, limitando as atividades de vida diária ${ }^{17}$.

Estudos apontam como a maior dificuldade na realização do CIL o não domínio da técnica pelos pacientes e/ou seus cuidadores. O posicionamento do paciente durante o procedimento, a adequada visualização do meato uretral, o conhecimento do método e da anatomia uretral permitem aos pacientes e seus cuidadores a sondagem uretral sem trauma e sem dor, evitando-se manobras que possam lesar a uretra ${ }^{16,18}$.

A dificuldade no manuseio dos dispositivos e na realização da técnica correta do CIL, como também a falta de orientação educativa prévia foram mencionados em pesquisa realizada envolvendo 11 pacientes que realizam o CIL para o esvaziamento da bexiga como obstáculos para a adesão ao procedimento de forma correta e segura ${ }^{19}$.

Embora as tecnologias em cateteres tenham avançado e tenham respaldo científico e o CIL seja o padrão ouro, a taxa de não adesão entre os pacientes permanece alarmantemente alta $^{2,4,10,20}$.

A não adesão pode levar a complicações sérias. É recomendável que o volume drenado não deve ultrapassar os $400 \mathrm{~mL}$ e uma frequência reduzida de cateterismos em 24 horas pode resultar em um maior risco de infecções urinárias, além da distensão da bexiga e risco de comprometimento renal a longo prazo ${ }^{20}$. 
É de fundamental importância que o enfermeiro especialista ou habilitado, execute a capacitação do paciente, familiares e cuidadores sobre a necessidade do cateterismo intermitente e a correta técnica de execução bem como os riscos inerentes à sua não realização e potenciais complicações ${ }^{20}$.

Dentre as limitações desta pesquisa, destacam-se o desenvolvimento do estudo com pacientes já cadastrados no serviço, portanto, com vícios próprios no procedimento, na preferência pelo dispositivo, e no uso dos cateteres envolvidos na avaliação, além do tempo disponível para a experimentação dos diferentes cateteres. Soma-se a isso, os erros no cadastro de pacientes, havendo perda de possíveis inclusões no estudo com aumento do número. Talvez a interpretação subjetiva que o paciente faz dos diferentes dispositivos necessite ser melhor explorada em estudos futuros incluindo pacientes recém cadastrados.

Os achados do trabalho mostram que os diferentes cateteres foram avaliados pelos pacientes envolvidos, sendo identificados tanto aspectos positivos como também negativos em relação a cada um deles. A preferência dos pacientes pelo cateter com revestimento hidrofílico de poliuretano foi confirmada, contudo temos que considerar as limitações deste trabalho e reconhecer que a maior parte dos pacientes já usava o referido dispositivo no momento da pesquisa.

Vale destacar que a tecnologia de preferência do atual estudo também foi a mesma incorporada em 2019 pela Comissão Nacional de Incorporação de Tecnologias (CONITEC) no SUS. E, também, no ano de 2020 houve uma tramitação para a publicação de um "Protocolo Clínico de Diretrizes Terapêuticas para Bexiga Neurogênica", reforçando ainda mais a necessidade e importância do tema em questão no cenário nacional ${ }^{21}$.

No Brasil, ainda não há política específica para garantir os materiais utilizados na realização do CIL. Contudo a população brasileira é assistida pelo SUS, que concebe saúde como direito fundamental do todo ser humano e atribui ao Estado o dever de proporcionar todas as condições fundamentais para seu pleno exercício, bem como garantir redução dos riscos de doenças e agravos. Nos princípios do SUS também é prevista a assistência terapêutica integral. Assim, a elaboração de políticas públicas que assegurem o acesso ao dispositivo de escolha do paciente para a execução do procedimento é imperativa ${ }^{9,22}$.

\section{CONCLUSÃO}

A satisfação dos usuários em relação à tecnologia deve estar aliada ao melhor atendimento das suas necessidades, e, neste estudo, identificou-se o cateter de poliuretano com revestimento hidrofilico o de maior preferência e atributos positivos quando comparado aos demais.

Por fim, considerou-se que a oportunidade dada aos pacientes com retenção urinária de conhecer, utilizar e avaliar os diferentes dispositivos foi benéfica, uma vez que deveria ser direito dos pacientes ter acesso a todas as tecnologias disponíveis no mercado e optar pela que melhor corresponda às suas necessidades e preferências.

Dessa forma, faz-se necessário o aprimoramento do conhecimento dos profissionais que indicam e orientam esse perfil de pacientes, além do desenvolvimento de políticas públicas de acesso à melhor tecnologia, favorecendo assim a adesão desses pacientes à realização da técnica correta, autonomia, redução dos riscos de complicações associadas ao procedimento, impactando diretamente em sua qualidade de vida.

\section{CONTRIBUIÇÃO DOS AUTORES}

Conceitualização: Bonello A; Metodologia: Bonello A e Mello MP; Investigação: Bonello A e Faci MC; Redação - Primeira versão: Bonello A, Faci MC e Mello MP; Redação - Revisão \& Edição: Bonello A, Faci MC e Mello MP. Recursos: Bonello A, Faci MC. Supervisão: Bonello A.

\section{DISPONIBILIDADE DE DADOS DE PESQUISA}

Todos os dados foram gerados ou analisados no presente estudo. 


\section{AGRADECIMENTOS}

A todas as empresas que contribuíram com as doações das tecnologias utilizadas no presente estudo.

\section{REFERÊNCIAS}

1. Assis GM, Faro ACM. Autocateterismo vesical intermitente na lesão medular. Rev Esc Enferm USP. 2011;45(1):289-93. https:// doi.org/10.1590/S0080-62342011000100041

2. Kennelly M, Thiruchelvam N, Averbeck MA, Konstatinidis C, Chartier-Kastler E, Trøjgaard P et al. Adult Neurogenic Lower Urinary Tract Dysfunction and Intermittent Catheterisation in a Community Setting: Risk Factors Model for Urinary Tract Infections. Adv Urol. 2019:1-13. https://doi.org/10.1155/2019/2757862

3. Rognoni C, Tarricone R. Intermittent catheterisation with hydrophilic and non-hydrophilic urinary catheters: systematic literature review and meta-analyses. BMC Urol. 2017;17:4. http://doi.org/10.1186/s12894-016-0191-1

4. Truzzi JC, Teich V, Pepe C. Cateteres com revestimento hidrofilico podem ser beneficos para o sistema público de saúde no Brasil? Uma análise de custo-efetividade em pacientes com lesão da medula espinhal. IBJU Int braz J urol. 2017;43. https://doi. org/10.1590/S1677-5538.IBJU.2017.0221

5. Assis GM, Fraga R. Cateterismo intermitente limpo: manual ilustrado de orientação ao usuário (adulto). Curitiba: Universidade Federal do paraná - Hospital de Clínicas, 2015. Disponível em: https://quemcuida.com.br/blog/wp-content/uploads/2016/01/ manual-cateterismo-vidas-secas.pdf

6. Lapides J, Diokno AC, Silber SJ, Lowe BS. Clean intermittent self-catheterization in the treatment of urinary tract disease. J Urol. 1972;107(3):45-61. https://doi.org/ 10.1016/s0022-5347(17)61055-3

7. Paula MAB, Thuler SR, Silveira NI, Azevedo GR. Intervenções nas Áreas de Abrangência da Estomaterapia. Lorena: CCTA, 2016. Disponível em: https://sobest.com.br/wp-content/uploads/2020/10/PDF_INTERVENCOES.pdf

8. Schoeller SD, Martins MM, Ribeiro I, Lima DKS, Padilha MICS, Gomes B. Breve Panorama Mundial da Enfermagem de Reabilitação. RPER. 2018;1(1):6-12. https://doi.org/10.33194/rper.2018.v1.n1.01.4388

9. Fumincelli L, Mazzo A, Martins JCA, Henriques FMD, Orlandin L. Qualidade de vida de pacientes usuários do cateterismo urinário intermitente. Rev Latino-Am Enfermagem. 2017;25:e2906. https://doi.org/10.1590/1518-8345.1816.2906

10. Norager R, Bogebjerg C, Plate I, Lemaitre S. Apoiando melhor a adesão entre os pacientes envolvidos em autocateterismo intermitente. Br J Nurs. 2019;28(2):90-5.

11. Assis GM, Negri AF, Veiga SA, Galli CF, Moser ADL, Azevedo GR. Elaboração e Validação de um Instrumento de Avaliação da Percepção do Usuário na Utilização de Cateteres Vesicais. ESTIMA, Brazilian J Enteros Ther. 2016;13(2):62-9. Disponível em: https://www.revistaestima.com.br/estima/article/view/105

12. Assis GM, Negri AF, Tung SAV, Saccomani CFG, Moser ADL, Azevedo GR et al. Uso de cateteres vesicais para cateterismo intermitente limpo: satisfação da pessoa com lesão medular. Cogitare Enferm. 2015;20(4):813-20. Disponível em: http:// revistas.ufpr.br/cogitare/article/view/41585

13. Pinder B, Lloyd AJ, Nafees B, Elkin EP, Marley J. Patient preferences and willingness to pay for innovations in intermittent selfcatheters. Patient Prefer Adherence. 2015;9:381-8. https://doi.org/10.2147/PPA.S73487

14. Feng D, Cheng L, Bai Y, Yang Y, Han P. Outcomes comparison of hydrophilic and non-hydrophilic catheters for patients with intermittent catheterization:An updated meta-analysis. AsianJSurg. 2020;43(5):633-5. https://doi.org/10.1016/j.asjsur.2019.12.009

15. DeFoor W, Reddy P, Reed M, VanderBrink B, Jackson E, Zhang B et al. Results of a prospective randomized control trial comparing hydrophilic to uncoated catheters in children with neurogenic bladder. J Pediatr Urol. 2017;13(4):373.e1-373.e5. https://doi.org/10.1016/j.jpurol.2017.06.003

16. Orlandin L, Nardi A, Costa RR de O, Mazzo A. Dificuldades de pacientes e cuidadores na realização do cateterismo intermitente limpo: revisão de escopo. ESTIMA, Brazilian J Enteros Ther. 2020;18:e1520. https://doi.org/10.30886/estima.v18.907_PT

17. Alencar VP. Cateterismo vesical intermitente limpo em crianças e adolescentes: análise da qualidade de vida do binômio cuidador-criança e dos fatores envolvidos. [tese]. São Paulo (SP): Faculdade de Medicina, Universidade de São Paulo; 2016. https://doi.org/10.11606/T.5.2016.tde-20092016-162358

18. Faleiros F, Pelosi G, Warschausky S, Tate D, Käppler C, Thomas E. Factors influencing the use of intermittent bladder catheterization by individuals with spina Bifida in Brazil and Germany. Rehabil Nurs. 2018;43(1):46-51. https://doi.org/10.1002/ rnj.302

19. Cobussen-Boekhorst H, Hermeling E, Heesakkers J, Gaal B. Patients' experience with intermittent catheterisation in everyday life. J Clin Nurs. 2016;25(9-10):1253-61. https://doi.org/10.1111/jocn.13146 
20. Truzzi JC, Canalini AF, Prezotti JA, Resplande J. Recomendações Sociedade Brasileira de Urologia 2016: Cateterismo Vesical Intermitente.

21. Comissão Nacional de Incorporação de Tecnologias no SUS. Cateter hidrofílico para cateterismo vesical intermitente em indivíduos com lesão medular e bexiga neurogênica. 2019. Disponível em: http://conitec.gov.br/images/Consultas/ Relatorios/2019/Sociedade/ReSoc140_SPEEDCATH_caterismo_vesical.pdf

22. Lei n. 8080 de 19 de Setembro de 1990 (BR). Dispõe sobre as condições para a promoção, proteção e recuperação da saúde, a organização e o funcionamento dos serviços correspondentes e dá outras providências. Diário Oficial da União [periódico na internet], Brasília (DF). 20 set 1990. Disponível em: https://legis.senado.leg.br/norma/549956 